\title{
Emendo - A Toolchain for Creating Gamified Learning Arrangements for Online Learning Settings
}

\author{
Bartel, Alexander ${ }^{\text {a }}$; Hagel, Georg ${ }^{\text {a }}$ and Wolff, Christian ${ }^{\text {b }}$ \\ ${ }^{a}$ Faculty of Computer Science, Kempten University of Applied Sciences, Germany, \\ ${ }^{\mathrm{b}}$ Faculty of Languages, Literature, and Cultures, University of Regensburg, Germany.
}

\begin{abstract}
This contribution describes the Emendo toolchain which enables the effective creation and implementation of gamified learning arrangements for online learning settings based on the domain-specific modeling approach. The components of Emendo are a domain-specific modeling language, a generator which transforms models based on the language into source code as well as the embedding of the latter in a learning management system. Scenarios for the usage of Emendo for teaching and learning are presented with respect to the functionalities of the toolchain. In addition, a qualitative evaluation concerning Emendo's goals, concept and insights on the results is given. The evaluation shows that Emendo reaches high acceptance for teaching purposes and can serve as a promising means for the digitisation of teaching and learning.
\end{abstract}

Keywords: Gamification; Learning; Learning Tool; Online Learning; Learning Management System, Domain-specific Modeling. 


\section{Introduction}

Learning and the associated acquisition of skills and competences is possible in many ways. One specific means is the use of online learning platforms or learning management systems (LMS). In order to enhance learning motivation as the central driving force and hence a prerequisite for learning to take place (Smolka, 2004), such platforms often integrate aspects of gamification. Recent studies show that the concept of gamification can successfully increase the learning motivation of individuals if it is integrated in learning management systems (e.g. Ibanez et al., 2014; Bartel \& Hagel, 2014; Hakulinen et al., 2015; Hasegawa et al., 2015). According to Deterding et al. (2011), gamification can be generically defined as "the use of game design elements in non-game contexts" (p. 10). In this contribution, gamification is considered in the context of learning, and we will use the following definition: "Gamification is described as a concept which integrates game design elements and processes into learning activities in order to increase learning motivation and thereby changes the behavior of learners" (Bartel \& Hagel, 2016, p. 6).

Although various reports exist on how to integrate aspects of gamification in existing LMS, e.g. as plugins into Moodle (Roderus, 2015) or as standalone applications (e.g. Hakulinen et al., 2015), researchers (Dicheva et al., 2014; Dicheva \& Dichev, 2016) argue that existing learning management systems that integrate aspects of gamification

- are not effective in bringing gamified learning arrangements into practice,

- don't provide an extensible set of elements for creating such arrangements and

- constrain instructors due to an inflexible environment.

\subsection{Goals}

This contribution demonstrates a novel toolchain called Emendo (Bartel et al., 2017) in order to overcome the issues mentioned above. Emendo can be effectively used for creating gamified learning arrangements in an online setting, it is extensible with regard to its elements and does not constrain instructors in their definition of gamified learning arrangements. Its basic toolchain structure as well as scenarios of usage for learning purposes are demonstrated. Furthermore an evaluation with lecturers is shown. This contribution complements the work described in Bartel et al. (2017) where Emendo was technically detailed.

\subsection{Structure}

The rest of this contribution is structured into three sections: The following section relates to the descriptions stated in Bartel et al. (2017) and presents an overview of the components of the Emendo toolchain. In addition, it shows some scenarios for using Emendo and its functionalities in learning contexts. The third section presents the goals, method and results 
of an evaluation conducted with lecturers using Emendo. The last section summarizes the findings and gives an outlook on future works.

\section{Emendo Toolchain}

\subsection{Overview}

The basic idea of Emendo was to build a system for creating and implementing gamified learning arrangements since researchers argue that there is a lack of tools which efficiently enable the creation of such arrangements for domain experts (Dicheva \& Dichev, 2017). For this pupose, the domain-specific modeling (DSM) approach (Kelly \& Tolvanen, 2008) was chosen because of the idea that domain experts without the need for technical knowledge should be able to use Emendo and bring their ideas to class. Figure 1 shows an overview of the Emendo toolchain and its components. As already stated in Bartel et al. (2017), currently there is no comparable approach in the educational field.

As documented in Bartel et al. (2017) and as a first step, a domain-specific modeling language (DSML) was created following a literature review considering more than 3600 papers and a domain analysis of 12 gamified learning platforms. More than 91 conceptual requirements (which were aggregated to concepts) for the Emendo DSML were elicitated including various types of: game design elements (e.g. Badges, Points, Levels, Leaderboards etc.), tasks (e.g. single choice, multiple choice etc.), learning materials (e.g. texts, videos, podcasts etc.), rules for rewarding game design elements and giving feedback, and elements to arrange them into learning paths. Subsequently the Emendo Designer was created which enables the creation of models of the Emendo DSML by domain experts. These models are instances of the abstract Emendo DSML and bring gamified learning arrangements into practice: A code generator processes the gamified learning arrangements and its output serves as input for the Emendo Learning Management System (LMS). The Emendo LMS allows learners to work with the learning arrangements defined by lecturers. During learners' interaction with Emendo, lecturers can interact with them by discussing questions and giving feedback on learners' answers. Furthermore lecturers can track the individual learning progress of learners with the use of learning analytics the LMS provides.

\subsection{Usage Scenarios}

Emendo allows various scenarios of usage, depending on educational goals and the context it is used in. This variety of applications is made possible by the design of the system: In contrast to existing learning management systems, Emendo does not aim to provide static heuristics for the modeling of gamified learning arrangements. 


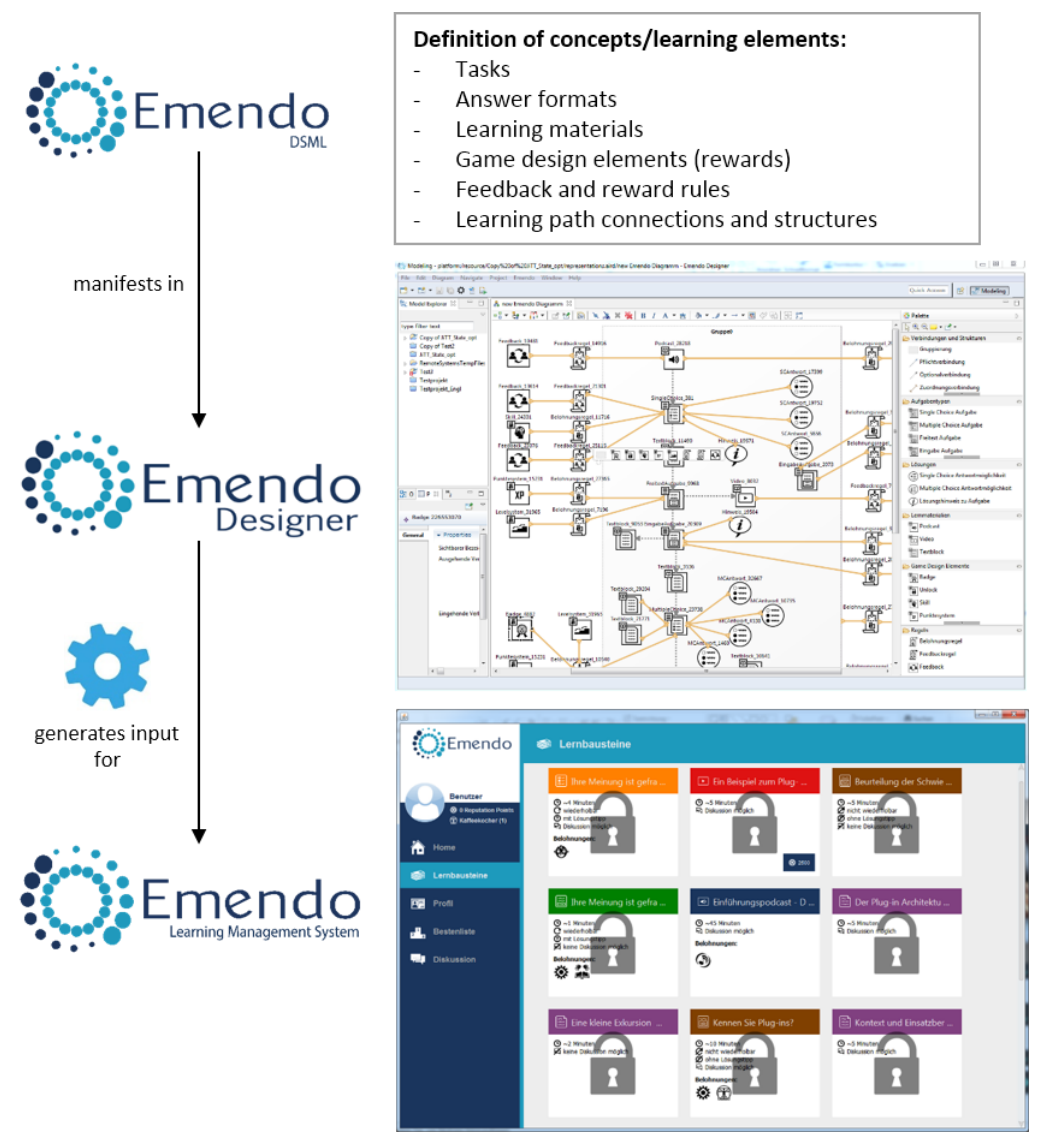

Figure 1. Overview of the Emendo toolchain

Emendo's basic heuristics can be changed and adapted as required and is not limited by a strict corset of building blocks for teaching. For example, if a particular type of learning task or a certain type of feedback is not represented, it can simply be added to the Emendo DSML as well as to the Emendo LMS. Admittedly, this adaptation requires a basic understanding of the technical functionality of Emendo. However, Emendo already provides a wide range of concepts that can be used in gamified online learning scenarios. In parts their use is described in the following from the perspective of lecturers.

Emendo allows the definition of individual learning paths and the structuring according to the learning goals or the educational contents of each path. This gives learners the freedom to choose which learning element they want to deal with according to their personal sense of competence and increases their sense of autonomy - an important influence on learning motivation according to the self-determination theory (Ryan \& Deci, 2000). Besides the structuring of learning paths, Emendo also distinguishes between learning elements that are 
optional and mandatory to process for learners. Hence, Emendo can be used simply for learning (e.g. reading texts, watching videos) or testing learners' performance (e.g. integrating a quiz consisting of different types of questions in the middle of a learning topic or in order to finalize a whole learning path). According to learners' actions and the progress state of learning elements, lecturers can integrate contextualized feedback. For example, if a learner answered a single choice question, Emendo allows the definition of several feedbacks, e.g. one which is displayed to provide a hint for its correct processing when it was answered incorrectly and another one which is shown when the answer was correct and to show its pedagogical value in the whole context (Nicholson, 2015). Besides feedback, learners can be rewarded with game design elements, like points or badges, which reflects typical elements of game mechanics like achievements or the collection of virtual goods (Werbach \& Hunter, 2015). The game design elements not only serve as an external incentive, but also as a means of determining relative progress in comparison with other learners. This leads to social inclusion and comparison which can further drive the motivation to learn (Nah et al., 2013).

Emendo is not only extensible regarding its concepts, it is also scalable in terms of learning units. An entire course covering a whole semester can be defined (e.g. as a blended learning course), as well as a single unit within a course (e.g. as a peer instruction unit). Furthermore, from a students' point of view, the LMS can either be used as a client on computers or mobile devices which allows formal learning in educational institutions and informal learning in their leisure time.

\section{Evaluation}

\subsection{Goals and Evaluation Concept}

A first evaluation was conducted that aimed at finding out how lecturers evaluate Emendo. The following research questions guided the evaluation:

- RQ1: How do lecturers judge Emendo regarding its applicability and usefulness for their teaching in general?

- $\quad$ RQ2: How do lecturers judge the modeling experience using the Emendo DSML in the Emendo Designer for creating a gamified learning arrangement?

To answer the research questions a two-part qualitative evaluation concept was developed: As a first step and in order to enhance comparability, a short video was shown to the test persons demonstrating the components and features of the Emendo toolchain, followed by an semi-standardized interview based on the Structure Laying Technique (SLT) according to Scheele \& Gröben (1988). In the second part, the test persons received a handout containing a description of the user interface of the Emendo Designer and explanations for the elements of the Emendo DSML. In addition, the handout contained a scenario 
describing a gamified learning arrangement as a learning unit for a software engineering course. After the guided application of the Emendo Designer, test persons were interviewed using the SLT. As in the first part of the evaluation, the SLT allows given patterns of actions to be combined with experiments. Additionally, all statements of probands were visualized with flashcards and were adjusted in dialogue with the researcher. Thus, the interviewer can ask for more information on a certain argument.

The sample of this qualitative evaluation includes $n=8$ ( 3 female, 5 male) university lecturers who participated voluntarily. Each evaluation had an average duration of 2 hours. To maximize variety, the persons interviewed were chosen according to specific personal criteria (e.g. experience in using gamification, specialist area, experience in teaching etc.). The interviews were recorded and analyzed together with the flashcards regarding the research questions.

\subsection{Results}

Due to capacity reasons only a small part of the results are demonstrated in this contribution. In general, both research questions can be answered positively. In particular, all participants stated that they would actually integrate Emendo in their teaching since it supports the quick implementation of ideas for teaching. The vast majority $(n=7)$ also stated that they see a high applicability of Emendo for learning because it is not bound to a technical discipline, its learning elements are extendable and it provides the possibility to increase students' learning motivation due to the use of gamification and the way it facilitates adaptive learning facets. Prerequisites for an integration in courses is a fast, intuitive and easy use of the tools, especially concerning the Emendo Designer with its DSML. Furthermore, the integration in existing LMS like Moodle has a high significance for the interview partners. In addition, Emendo needs to provide a solid documentation and support (e.g. automated imports) to keep the effort for switching over from existing course compositions low. Regarding the second research question, figure 2 gives an overview of the aggregated positive (green) and negative (red) statements.

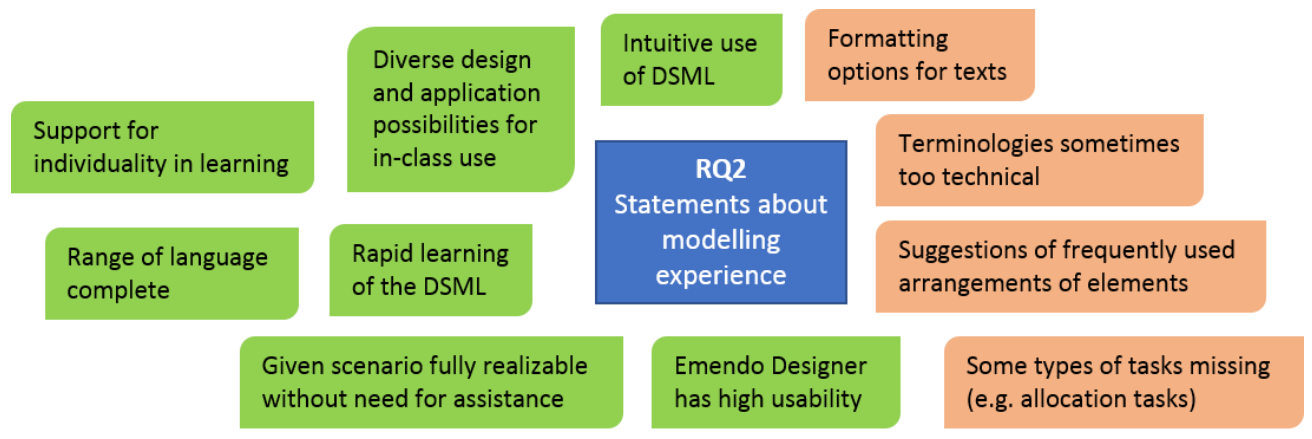

Figure 2. Aggregated result statements regarding $R Q 2$ 


\section{Conclusion and Future Work}

This contribution demonstrates the Emendo toolchain, its components and suggestions for scenarios of usage based on its functionalities. A qualitative evaluation of its goals, concepts is shown along with insights on results. Emendo introduces a new type of platform-based and gamified online learning that reaches high acceptance for teaching purposes. However, the evaluation also reveals some issues for improvement. Besides that, another evaluation focusing on learners' needs will be conducted as a next step in order to examine learners' attitudes towards the LMS and this specific way of learning. After that, more studies can follow which should be conducted in different subject areas, but at the same time using comparable designs of gamified learning arrangements.

\section{References}

Bartel, A., Hagel, G., \& Wolff, C. (2017). Effective Integration of Gamification and Learning Management Systems for Creating Gamified Learning Arrangements. In J. Domenech, C. M. Vicent-Vela, E. de La Poza, \& D. Blazquez (Eds.), Proceedings of the 3rd International Conference on Higher Education Advances (pp. 679-686).

Bartel, A., \& Hagel, G. (2014). Engaging Students With a Mobile Game-Based Learning System in University Education. International Journal of Interactive Mobile Technologies, 8(4), 56-60.

Bartel, A., \& Hagel, G. (2016). Gamified Just-in-Time Teaching - A Conceptual Approach Based on Best Practices. In G. Hagel \& J. Mottok (Eds.), Proceedings of ECSEE 2016 European Conference Software Engineering Education (pp. 1-17). Aachen: Shaker.

Deterding, S., Dixon, D., Khaled, R., \& Nacke, L. (2011). From game design elements to gamefulness: defining gamification. In A. Lugmayr, H. Franssila, C. Safran, \& I. Hammouda (Eds.), Proceedings of the 15th International Academic MindTrek Conference: Envisioning Future Media Environments (pp. 9-15).

Dicheva, D., \& Dichev, C. (2016). An Active Learning Model Employing Flipped Learning and Gamification Strategies. In Proceedings of first Int. Workshop on Intelligent Mentoring Systems (IMS 2016), Zagreb, Croatia (pp. 1-6).

Dicheva, D., Irwin, K., Dichev, C., \& Talasila, S. (2014). A course gamification platform supporting student motivation and engagement. In 2014 International Conference on Web \& Open Access to Learning (ICWOAL) (pp. 1-4). Piscataway, NJ: IEEE.

Hakulinen, L., Auvinen, T., \& Korhonen, A. (2015). The Effect of Achievement Badges on Students' Behavior: An Empirical Study in a University-Level Computer Science Course. International Journal of Emerging Technologies in Learning (iJET), 10(1), 1829. 
Hasegawa, T., Koshino, M., \& Ban, H. (2015). An English vocabulary learning support system for the learner's sustainable motivation. SpringerPlus, 4(99).

Ibanez, M., Di Serio, A., \& Delgado Kloos, C. (2014). Gamification for Engaging Computer Science Students in Learning Activities: A Case Study. IEEE Transactions on Learning Technologies, 7(3), 291-301.

Kelly, S., \& Tolvanen, J.-P. (2008). Domain-specific modeling: Enabling full code generation. Hoboken, N.J.: John Wiley \& Sons.

Nah, F. F.-H., Telaprolu, V. R., Rallapalli, S., \& Venkata, P. R. (2013). Gamification of Education Using Computer Games. In S. Yamamoto (Ed.), Lecture Notes in Computer Science. Human Interface and the Management of Information. Information and Interaction for Learning, Culture, Collaboration and Business (Vol. 8018, pp. 99-107). Springer Berlin Heidelberg.

Nah, F. F.-H., Zeng, Q., Telaprolu, V. R., Ayyappa, A. P., \& Eschenbrenner, B. (2014). Gamification of Education: A Review of Literature. In F. F.-H. Nah (Ed.), HCI in Business: First International Conference, HCIB 2014, Heraklion, Crete, Greece, June 22-27, 2014. Proceedings (pp. 401-409). Cham: Springer.

Nicholson, S. (2015). A RECIPE for Meaningful Gamification. In T. Reiners \& L. C. Wood (Eds.), Gamification in Education and Business (pp. 1-20). Cham: Springer International Publishing.

Roderus, S. (2015). Ein Kurskonzept zur Förderung des kontinuierlichen Lernens durch den Einsatz von Gamification. In Zentrum für Hochschuldidaktik (Ed.), Tagungsband zum 2. HDMINT Symposium 2015 (pp. 81-86).

Ryan, R. M., \& Deci, E. L. (2000). Self-Determination Theory and the Facilitation of Intrinsic Motivation, Social Development, and Well-Being. American Psychologist, $55(1), 68-78$.

Scheele, B., \& Groeben, N. (1988). Dialog-Konsens-Methoden zur Rekonstruktion subjektiver Theorien: D. Heidelberger Struktur-Lege-Technik (SLT), konsensuale ZielMittel- Argumentation $u$. kommunikative Flußdiagramm-Beschreibung von Handlungen. Tübingen: Francke.

Smolka, D. (2004). Schülermotivation: Konzepte und Anregungen für die Praxis (2., durchges. Aufl). München, Neuwied: Luchterhand.

Werbach, K., \& Hunter, D. (2015). The Gamification Toolkit: Dynamics, Mechanics, and Components for the Win. New York: Wharton Digital Press. 